\title{
ITERATED CLASS FORCING
}

\author{
SY D. FRIEDMAN
}

In this paper we develop the notion of "stratified" class forcing and show that this property both implies cofinality-preservation and is preserved by iterations with the appropriate support. Many Easton-style and Jensen-style forcings are stratified, as are some more exotic forcings obtained by mixing these types together (see Easton [70], section 36 of Jech [78], Beller-Jensen-Welch [82], Friedman [90]).

As a sample application, cofinalities are preserved by an iteration of length ORD where at even stages $i$, an Easton-style forcing adds a Cohen set to regular cardinals $\geq$ card $(i)$, at odd stages $i+1$ the class added at stage $i$ is coded by a subset of the least infinite regular cardinal $\geq$ card(i) via the techniques of Friedman [94A] or Friedman [94B], and for any regular $\kappa,\{i \mid p(i)$ is nontrivial below $\kappa\}$ is a subset of $\kappa^{+}$of size $<\kappa$, for each condition $p$ in the iteration.

Jensen coding as in Beller-Jensen-Welch [82] is not stratified but obeys a related property, called $\Delta$-stratification, which is also preserved by iterations with the appropriate (larger) support. As a sample application, the original form of Jensen coding can be used in the iteration of the preceding paragraph, provided the Cohen sets are added at successor cardinals only, full support is used and the condition stated at the end of that paragraph is imposed only at successor cardinals.

We now define stratification, in the language of Gödel-Bernays class theory.

Definition. $P$ (partially ordered by $\leq$ ) is stratified if there is a class $A$ such that $V=L[A],\langle V, A\rangle$ has a $A$-definable well ordering and:

(a) $P$ and $\leq$ are $A$-definable. A condition in $P$ is a function $p$ on an initial segment of Card $=\{0\} \cup$ Infinite Cardinals, where if $q$ extends $p$ as a function, $q(\gamma)=\emptyset$ for all $\gamma \in \operatorname{Dom}(q)-\operatorname{Dom}(p)$, then we identify $p$ with $q$. Also we require that $p(\kappa)=\emptyset$ for singular $\kappa$ and the conditions with

Research supported by NSF Grant DMS-9205530.

Received November 4, 1993. 
constant value $\emptyset$ are the weakest in $P$. Lastly, $\left\{p \mid p\left\lceil\kappa^{+} \in H_{\kappa^{+}}\right\}\right.$is dense for each $\kappa \in$ Card.

(b) ( $\kappa$-Density Reduction) Let $\kappa$ be regular and define $p \leq_{\kappa} q$ if $\left(p \leq q\right.$ and $p\left\lceil\kappa^{+}=q\left\lceil\kappa^{+}\right)\right.$. Then $p \leq q \longrightarrow \exists r \leq_{\kappa} q \exists s \leq r\left(s \uparrow\left[\kappa^{+}, \infty\right)=\right.$ $r \uparrow\left[\kappa^{+}, \infty\right)$ and $\left.s \leq p\right)$ and $\exists r \exists s\left(p \leq r, p \leq s\right.$ and $r \uparrow \kappa^{+}=q \uparrow \kappa^{+}$, $\left.s \uparrow\left[\kappa^{+}, \infty\right)=q \uparrow\left[\kappa^{+}, \infty\right)\right) \longrightarrow p \leq q$. If $D$ is an $A$-definable dense class, $p$ a condition then $\exists q \leq_{\kappa} p \exists d \subseteq D(\operatorname{card}(d) \leq \kappa$ and every $r \leq q$ can be extended to $s$ such that $s \uparrow\left[\kappa^{+}, \infty\right)=r \uparrow\left[\kappa^{+}, \infty\right)$ and $s$ extends an element of $d)$.

(c) ( $\kappa$-Definable Closure) For infinite regular $\kappa$ there are $\prod_{\sim}^{A}$ operators $F_{n}(x, \kappa, p)$ for $0<n \in \omega$ such that $F_{n}(x, \kappa, p) \leq_{\kappa} p$ for all $p$ and whenever $p_{0} \geq_{\kappa} p_{1} \geq_{\kappa} \ldots$ is a $\Pi_{n}^{A}$ (in parameters from $\kappa \cup\{x\}$ ) sequence of length $\lambda \leq \kappa$ such that for each $i<\lambda, p_{i+1} \leq_{\kappa_{i}} F_{n}\left(x, \kappa_{i}, p_{i}^{\prime}\right)$ for some $p_{i}^{\prime} \leq_{\kappa_{i}} p_{i}$ and regular $\kappa_{i} \geq \kappa$ then there is a single $p$ s.t. $p \leq_{\kappa} p_{i}$ for all $i<\lambda$.

Remark. $\kappa$-Density Reduction is at the heart of the usual proofs that when forcing notions are "factored," it is forced by the trivial condition of the "upper part" that the "lower part" has the appropriate chain condition. We shall find it convenient to make use of the following definition: $q$ reduces $D$ below $\kappa^{+}$to size $\leq \kappa$ iff $\exists d \subseteq D$ (card $(d) \leq \kappa$ and every $r \leq q$ can be extended to $s$ s.t. $s \uparrow\left[\kappa^{+}, \infty\right)=r \uparrow\left[\kappa^{+}, \infty\right)$ and $s$ extends an element of d).

Theorem 1. Suppose that $P$ is stratified. Then $P$ preserves $Z F C$ (relative to the class A witnessing stratification), cofinalities and the GCH.

Proof. Using $\kappa$-Density Reduction repeatedly and $\kappa$-Definable Closure at the end, we get: If $\left\langle D_{i} \mid i<\kappa\right\rangle$ is a sequence of dense classes with $D_{i} A$ definable uniformly in $i$ and $p \in P$ then there is $q \leq p$ and $d$ of cardinality $\leq \kappa$ such that each $r \leq q$ is compatible with an element of $D_{i} \cap d$, for each $i$. $d$ will be the union of the $d_{i}$, where $d_{i}$ is obtained from $\kappa$-Density Reduction at stage $i$ applied to $D_{i}$. This implies that the forcing relation for $\underset{\sim}{\Delta_{0}^{A}}$ sentences is $A$-definable and that $A$-replacement, cofinalities are preserved.

To show that GCH is preserved let $p \Vdash \tau \subseteq \kappa$, where $\kappa$ is an infinite cardinal and define $D_{i}=\{q \mid q$ is incompatible with $p$ or $q \Vdash i \in \tau$ or $q \Vdash i \notin \tau\}$. Then $D_{i}$ is dense and $A$-definable, uniformly in $i<\kappa$. First suppose that $\kappa$ is regular. Then by $\kappa$-Density Reduction and $\kappa$-Definable Closure there is $q \leq p$ and $d$ of size $\leq \kappa$ such that $r \in d \longrightarrow r \uparrow\left[\kappa^{+}, \infty\right)=$ $q \uparrow\left[\kappa^{+}, \infty\right), r \uparrow \kappa^{+} \in H_{\kappa^{+}}$and $\{r \in d \mid r \Vdash i \in \tau$ or $r \Vdash i \notin \tau\}$ is predense below $q$ for each $i<\kappa$. ( $X$ is predense below $q$ if every extension of $q$ is compatible with an element of $X$.) Thus in the generic extension each 
subset of $\kappa$ is determined by a $\kappa$-sequence of size $\leq \kappa$ subsets of $H_{\kappa^{+}}$of the ground model, so the property $2^{\kappa}=\kappa^{+}$is preserved.

When $\kappa$ is singular the above argument can be repeated, using $\kappa_{i^{-}}$ Density Reduction and $\operatorname{cof}(\kappa)$-Definable closure, where $\left\langle\kappa_{i} \mid i<\operatorname{cof}(\kappa)\right\rangle$ is a sequence of regular cardinals converging to $\kappa$.

To preserve stratification under iteration we must discuss strong witnesses and diagonal supports.

Definition. $P$ is stratified with strong witness $A$ if in the definition of stratified, $V=L[A]$ and $\langle V, A\rangle$ has a $\Delta_{1}$-definable well-ordering, $P, \leq$, Card are $\underset{\sim}{\Delta_{1}^{A}}$ and there is a $\underset{\sim}{\Sigma_{1}^{A}}$ function $\underset{f}{f}(x, \kappa, p)=(q, d)$ such that if $x$ is an index for a $\sum_{\sim}^{A}$ dense class $D, p$ a condition and $\kappa$ infinite and regular then $q \leq_{\kappa} p$, card $(d) \leq \kappa, d \subseteq D$ and every $r \leq q$ can be extended to $s$ such that $s \uparrow\left[\kappa^{+}, \infty\right)=r \uparrow\left[\kappa^{+}, \infty\right)$ and $s$ extends an element of $d$.

Proposition 2. If $P$ is stratified then $P$ has a strong witness.

Proof. Suppose $A$ witnesses that $P$ is stratified. Then we can choose $A^{*}=$ $\sum_{N}^{A}$ satisfaction, for a large $N$ so that $V$ has a $\underset{\sim}{\Delta_{1}^{A^{*}}}$ well ordering and $P, \leq$, Card, $\{(q, d, \kappa) \mid \operatorname{card}(d) \leq \kappa$ and every $r \leq q$ can be extended to $s$ such that $r \uparrow\left[\kappa^{+}, \infty\right)=s \uparrow\left[\kappa^{+}, \infty\right)$ and $s$ extends an element of $\left.d\right\}$ are $\Delta_{\sim}^{A^{*}}$. Then the desired $\underset{\sim}{\Sigma_{1}^{A}}$ function $f(x, \kappa, p)$ exists. Finally define the $\prod_{\sim}^{A^{*}}$ operator $F_{n}^{*}(x, \kappa, p)$ to be $F_{N+n}(x, \kappa, p)$ where $\left\langle F_{m}(x, \kappa, p) \mid 0<m \in \omega\right\rangle$ comes from $A$.

Strong witnesses help us control the definability of the forcing relation.

Theorem 3. Suppose that $A$ is a strong witness to the stratification of $P$. Then the forcing relation for $P$ restricted to $\underset{\sim}{\Sigma_{1}^{A}}$ sentences is densely- $\sum_{\sim}^{A}$ : there is a $\sum_{\sim}^{A}$ relation $p \stackrel{*}{\Vdash} \varphi\left(p \in P, \varphi \underset{\sim}{\sum_{1}^{A}}\right)$ such that $p \stackrel{*}{\Vdash} \varphi \longrightarrow p \Vdash \varphi$ and $p \Vdash \varphi \longrightarrow \exists q \leq p, q \stackrel{*}{\Vdash} \varphi$.

Proof. It suffices to prove this for ${\underset{\sim}{0}}_{0} \varphi$, by looking at witnesses. We show by a $\Sigma_{1}^{A}$ induction on $\varphi$ that given $p$ we can (in a $\Sigma_{1}^{A}$ way) find $q \leq p$ and $i \in\{0,1\}$ such that either $q \Vdash \varphi, i=0$ or $q \Vdash \neg \varphi, i=1$. This will prove the Theorem since we can then take $q \stackrel{*}{\Vdash} \varphi \longleftrightarrow$ For some $p,(q, 0)$ arises from $p, \varphi$ as above.

The interesting case of the induction is the bounded quantifier: Suppose $\varphi$ is $\forall x \in \sigma \psi(x)$ where $\sigma$ is a term of rank $\alpha$. By induction we can effectively extend $p$ to decide any instance $\psi(\tau), \operatorname{rank}(\tau)<\alpha$. If one of 
these extensions $q_{\tau}$ forces $\tau \in \sigma \wedge \neg \psi(\tau)$ then we can take the desired $q$ to be $q_{\tau} \Vdash \neg \varphi$. Otherwise, we can build uniformly ${\underset{\sim}{1}}_{1}^{A}$ dense classes $D_{\tau}$, $\operatorname{rank}(\tau)<\alpha$ of conditions forcing $\tau \in \sigma \longrightarrow \psi(\tau)$. As $A$ is a strong witness we can effectively find $d$ and $q \leq p$ such that each $D_{\tau} \cap d$ is predense below $q$, where $d$ is a set. But this $q$ forces $\forall x \in \sigma \psi(x)=\varphi$.

Remark. By replacing $A$ by $\Sigma_{1}^{A}$-satisfaction in Theorem 3, we get that $\Vdash$

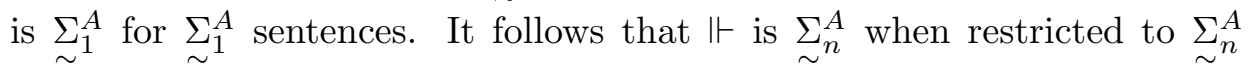
sentences. Also note, for later use, that Theorem 3 only requires that (b), (c) in the definition of stratification hold at cofinally many regular cardinals.

We are ready to discuss stratified iterations.

Definition. $\left\langle P_{i} \mid i<\alpha\right\rangle$ (where $P_{i+1}=P_{i} * \mathbb{Q}_{i}, P_{\lambda} \subseteq$ Inverse Limit $\left\langle P_{i}\right| i<$ $\lambda\rangle$ for limit $\lambda$ ) is a stratified iteration if for some class $A \subseteq O R D, A$ strongly witnesses that $P_{0}$ is stratified, for each $i+1<\alpha, \emptyset_{i} \Vdash_{i} \mathbb{Q}_{i}$ is stratified with strong witness $\left\langle A, G_{i}\right\rangle$ via some $F_{n}^{i}, f^{i}\left(\emptyset_{i}=\right.$ weakest condition in $P_{i}, \Vdash_{i}=$ forcing for $P_{i}, G_{i}=$ generic for $\left.P_{i}\right)$ and $\mathbb{Q}_{i}, f^{i}$ are $\Delta_{\sim}^{A},_{i}$ uniformly in $i<\alpha$, $F_{n}^{i}$ is $\prod_{\sim}^{A, G_{i}}$ uniformly in $i<\alpha$, for each $n>0$. Such an iteration has short diagonal supports if for $j<\alpha, p \in P_{j}$ and infinite regular $\kappa,\{i \mid i<j$ and $p\left\lceil i \forall_{i} \forall \gamma<\kappa^{+}, p(i)(\gamma)=\emptyset\right\}$ is a subset of $\kappa^{+}$of size $<\kappa$ (and this is the only restriction on supports).

Stratification Theorem. Suppose $\left\langle P_{i}\right| i\langle\alpha\rangle$ is a stratified iteration with short diagonal supports and GCH holds. Then $P_{\alpha}$ is definably isomorphic to a stratified forcing (relative to a class A witnessing stratification).

Proof. First we note that in the definition of stratified, we may assume one further condition about the operators $F_{n}(x, \kappa, p): \kappa_{1} \leq \kappa_{2}$ both regular, $p(\gamma)=\emptyset$ for all $\gamma<\kappa_{2} \longrightarrow F_{n}\left(x, \kappa_{1}, p\right)=F_{n}\left(x, \kappa_{2}, p\right)$. For, we may achieve this property by redefining $F_{n}$ to be $F_{n}^{*}(x, \kappa, p)=F_{n}(x, \kappa(p), p)$ where $\kappa(p)=$ least $\gamma$ such that $p(\gamma) \neq \emptyset$, if $\kappa \leq \kappa(p)$ and $F_{n}^{*}(x, \kappa, p)=F_{n}(x, \kappa, p)$ otherwise.

We prove the Main Theorem by induction on $\alpha$, maintaining the coherence property that the isomorphism of $P_{\alpha}$ with a stratified forcing $P_{\alpha}^{*}$ extend the (inductively produced) isomorphism of $P_{\beta}$ with the stratified forcing $P_{\beta}^{*}$ for $\beta<\alpha$, viewing $P_{\beta}$ as a subforcing of $P_{\alpha}$ in the natural way (and $P_{\beta}^{*}$ as a subforcing of $P_{\alpha}^{*}$ in a natural way that will be evident from the construction). Also if $A$ is our given witness to the stratification of the iteration then $A$ will serve as a strong witness to the stratification of each $P_{\alpha}^{*}$. 
The result is vacuous for $\alpha=0$ or 1 . Suppose that $\alpha=\beta+1$ is a successor ordinal $>1$. By induction $P_{\beta}$ is isomorphic to a stratified forcing $P_{\beta}^{*}$ and let $\leq_{\kappa}^{\beta}, F_{n}^{\beta}\left(x, \kappa, p^{\beta}\right)$ come from the stratification of $P_{\beta}^{*}$. Also $\emptyset_{\beta} \Vdash_{\beta} \mathbb{Q}_{\beta}^{*}$ is stratified $\left(\emptyset_{\beta}=\right.$ weakest condition of $P_{\beta}^{*}, \Vdash_{\beta}=$ forcing for $P_{\beta}^{*}, \mathbb{Q}_{\beta}^{*}=$ the $P_{\beta}^{*}$-name for $\left.\mathbb{Q}^{\beta}\right)$ and let $\leq_{\kappa}, F_{n}(x, \kappa, q)$ result from this.

By the Remark after Theorem 3, we may assume that $\Vdash_{\beta}$ is $\sum_{n}^{A}$ when

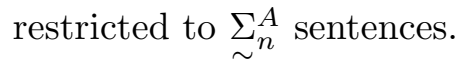

Now we define $P_{\alpha}^{*}$ to essentially consist of all functions $f$ on an initial segment of Card such that for some $p^{\beta}$ in $P_{\beta}^{*}$ and some $q, p^{\beta} \Vdash_{\beta} q \in \mathbb{Q}_{\beta}^{*}$ and for all $\kappa \in \operatorname{Dom}(f), f(\kappa)=\left\langle p^{\beta}(\kappa), q(\kappa)\right\rangle$ where $q(\kappa)$ is the canonical term denoting the result of applying to $\kappa$ the function named by $q$. However we must make two small modifications: insist that if $p^{\beta}(\kappa)=\emptyset$ and $p^{\beta} \Vdash_{\beta}$ $q(\kappa)=\emptyset$ or undefined then $f(\kappa)=\emptyset$ (instead of $\langle\emptyset$, a term for $\emptyset\rangle$ ); also insist that $\operatorname{Dom}(f)$ contains $\operatorname{Dom}\left(p^{\beta}\right)$ and $\operatorname{rank}(q)<\cup \operatorname{Dom}(f)$, so that $\emptyset_{\beta}$ $\Vdash_{\beta} \operatorname{Dom}(q) \subseteq \operatorname{Dom}(f)$. Then clearly $P_{\alpha}^{*}$ is isomorphic to $P_{\alpha}$ when $P_{\alpha}^{*}$ is ordered in the natural way (by ordering the corresponding pairs $\left\langle p^{\beta}, q\right\rangle$ in $P_{\beta}^{*} * \mathbb{Q}_{\beta}^{*}$ ). It is easy to verify condition (a) and the first part of (b) in the definition of stratification.

Next we demonstrate $\kappa$-Density Reduction for $P_{\alpha}^{*}$. For notational purposes we think of a condition in $P_{\alpha}^{*}$ as an element of $P_{\beta}^{*} * \mathbb{Q}_{\beta}^{*}$ (isomorphic to $P_{\alpha}^{*}$. Suppose $D \subseteq P_{\alpha}^{*}$ is dense and $A$-definable and $\left(p^{\beta}, q\right) \in P_{\alpha}^{*}$. Consider $D^{G_{\beta}^{*}}=\left\{q_{0} \in \mathbb{Q}_{\beta}^{*} \mid\left(p_{0}^{\beta}, q_{0}\right) \in D\right.$ for some $\left.p_{0}^{\beta} \in G_{\beta}^{*}\right\}$ where $G_{\beta}^{*}$ denotes the $P_{\beta}^{*}$-generic. Then $D^{G_{\beta}^{*}} \subseteq \mathbb{Q}_{\beta}^{*}$ is forced by $\emptyset_{\beta}^{*} \in P_{\beta}^{*}$ to be dense. So by $\kappa$-Density Reduction for $\mathbb{Q}_{\beta}^{*}, \emptyset_{\beta}^{*}$ also forces that $\left\{q_{0} \in \mathbb{Q}_{\beta}^{*} \mid q_{0}\right.$ reduces $D^{G_{\beta}^{*}}$ below $\kappa^{+}$, to size $\left.\leq \kappa\right\}$ is $\leq_{\kappa}$-dense on $\mathbb{Q}_{\beta}^{*}$. Thus $D_{\beta}=\left\{p_{0}^{\beta} \mid\right.$ For some $q_{0}, d_{0}, p_{0}^{\beta} \Vdash_{\beta} q \notin \mathbb{Q}_{\beta}^{*}$ or $q_{0} \leq_{\kappa} q$ reduces $D^{G_{\beta}^{*}}$ below $\kappa$, to $d_{0}$, card $\left.\left(d_{0}\right) \leq \kappa\right\}$ is dense on $P_{\beta}^{*}$. Let $p_{0}^{\beta} \leq_{\kappa}^{\beta} p^{\beta}$ reduce $D_{\beta}$ below $\kappa^{+}$, to size $\leq \kappa$, by $\kappa$-Density Reduction for $P_{\beta}^{*}$.

Then we can form terms $q_{0}, d_{0}$ such that $p_{0}^{\beta} \Vdash_{\beta} q_{0} \leq_{\kappa} q, q_{0}$ reduces $D^{G_{\beta}^{*}}$ below $\kappa^{+}$, to $d_{0}$ of size $\leq_{\kappa}$. For each $i<\kappa$ it is dense below $p_{0}^{\beta}$ to force some $p_{0}^{\beta}(i) \in G_{\beta}^{*},\left(p_{0}^{\beta}(i), d_{0}(i)\right) \in D$, where $d_{0}(i)=i^{\text {th }}$ element of $d_{0}$. Finally, by $\kappa$-Density Reduction and $\kappa$-Definable Closure for $P_{\beta}^{*}$, we can assume that $p_{0}^{\beta}$ reduces all of these dense sets below $\kappa^{+}$, to size $\leq \kappa$ and hence $\left(p_{0}^{\beta}, q_{0}\right)$ reduces $D$ below $\kappa^{+}$, to size $\leq \kappa$.

To complete the successor case we need to define the operators $F_{n}^{\beta+1}(x$, $\left.\kappa,\left(p^{\beta}, q\right)\right)$ and verify condition (c) in the definition of stratified. We set $F_{n}^{\beta+1}\left(x, \kappa,\left(p^{\beta}, q\right)\right)=$ "least" $\left(p_{0}^{\beta}, q_{0}\right)$ such that $p_{0}^{\beta} \leq_{\kappa}^{\beta} F_{n}^{\beta}\left(x, \kappa, p^{\beta}\right)$ and $p_{0}^{\beta} \Vdash_{\beta} F_{n}(x, \kappa, q)=q_{0}$. Note that the property of $\left(p_{0}, q_{0}\right)$ stated here 
is $\sum_{\sim}^{A}$ (in the other parameters), so we take "least" in the sense of $\sum_{\sim}^{A}$ -

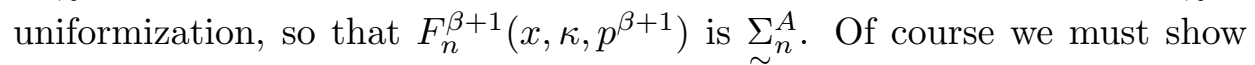
that such a $\left(p_{0}^{\beta}, q_{0}\right)$ exists. Note that it is a dense property of $p_{0}^{\beta}$ to force a value for $F_{n}(x, \kappa, q)$. By $\kappa$-Density Reduction there is $p_{0}^{\beta}$ reducing this dense property to a set, with $p_{0}^{\beta} \leq_{\kappa}^{\beta} F_{n}^{\beta}\left(x, \kappa, p^{\beta}\right)$. Thus we can form a term $q_{0}$ such that $p_{0}^{\beta} \Vdash_{\beta} F_{n}(x, \kappa, q)=q_{0}$.

The $\kappa$-Definable Closure of $P_{\beta+1}^{*}$ follows from the $\kappa$-Definable Closure of $P_{\beta}^{*}$ (relative to $A$ ) and the $\kappa$-Definable Closure of $\mathbb{Q}_{\beta}^{*}$ (relative to

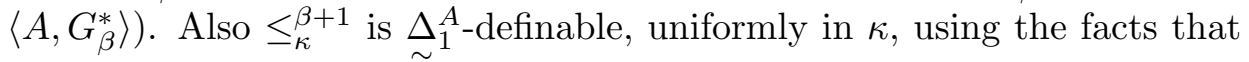
$\leq_{\kappa}^{\beta}$ is uniformly $\underset{\sim}{\Delta_{1}^{A}}, \leq_{\kappa}$ is uniformly $\underset{\sim}{\Delta}{ }_{1}^{A, G_{\beta}}$ and the fact that $\Vdash_{\beta}$ is $\underset{\sim}{\Sigma_{1}^{A}}$ when restricted to $\Sigma_{1}$ sentences.

Now we turn to the case where $\alpha$ is a limit ordinal. We take $P_{\alpha}^{*}$ to consist of all functions $f$ on an initial segment of Card such that for some $\left\langle f_{\beta} \mid \beta<\alpha\right\rangle$ in the short-diagonal support limit of $\left\langle P_{\beta}^{*} \mid \beta<\alpha\right\rangle$, $f(\kappa)=\left\langle f_{\beta}(\kappa) \mid \beta<\alpha\right\rangle$ for all $\kappa$ in $\operatorname{Dom}(f)$; we also require that $\operatorname{Dom}(f) \supseteq$ $\operatorname{Dom}\left(f_{\beta}\right)$ for each $\beta<\alpha$ and modify $f(\kappa)$ to be $\emptyset$ if $f_{\beta}(\kappa)=\emptyset$ for all $\beta<\alpha$. The $f$ 's are ordered by ordering the corresponding $\left\langle f_{\beta} \mid \beta<\alpha\right\rangle$ 's.

We must show that $\left\{f \in P_{\alpha}^{*} \mid f \uparrow \kappa^{+} \in H_{\kappa^{+}}\right\}$is dense for each $\kappa$. We actually show a bit more, for the purpose of carrying out an inductive argument: if $\gamma<\kappa$ belong to Card, $\gamma$ regular then $\left\{f \in P_{\alpha}^{*} \mid f \uparrow[\gamma, \kappa] \in\right.$ $\left.H_{\kappa^{+}}\right\}$is $\leq_{\gamma^{-}}$dense (i.e., any $f$ can be $\leq_{\gamma^{-}}$extended into this set; for $\gamma=0$ take $\leq_{\gamma}=\leq$.) Note that this stronger version follows from the weaker one, given $\gamma$-Density Reduction, so we may inductively assume that it holds for $P_{\beta}^{*}, \beta<\alpha$. Now we induct on $\kappa$ : using short diagonal supports, we may assume that $\operatorname{cof}(\alpha)<\kappa$ as otherwise our given $f$ has the property that for some $\beta_{0}<\alpha, f_{\beta}$ is the $\emptyset$-function below $\kappa^{+}$for all $\beta_{0} \leq \beta<\alpha$ (where $f$ comes from $\left\langle f_{\beta} \mid \beta<\alpha\right\rangle$ ) and so we can apply induction at $\beta_{0}$. By induction on $\kappa$ we can first extend $f$ to guarantee that $f \uparrow[\gamma, \operatorname{cof}(\alpha))$ belongs to $H_{\operatorname{cof}(\alpha)^{+}}$. So we may assume that $\gamma \geq \operatorname{cof}(\alpha)$. Now, choose a cofinal $\operatorname{cof}(\alpha)$-sequence $\alpha_{0}<\alpha_{1}<\ldots$ below $\alpha$ and successively extend $f=f_{0}$ to $f_{1}, f_{2}, \ldots$ in $\operatorname{cof}(\alpha)$ steps so that $\left(f_{i+1}\left\lceil\alpha_{i}\right)\right.$ on $[\gamma, \kappa]$ belongs to $H_{\kappa^{+}}$and $f_{i+1}\left\lceil\alpha_{j} \geq_{\gamma} F_{1}^{\alpha_{j}}\left(x, \gamma, f_{i} \uparrow \alpha_{j}\right)\right.$ for all $j \leq i$, where $F_{1}^{\alpha_{i}}$ comes from Definable Closure for $P_{\alpha_{i}}$ and $x=\left\langle f, \gamma, \kappa,\left\langle\alpha_{i} \mid i<\operatorname{cof} \alpha\right\rangle\right\rangle$. (We abuse notation slightly; $f_{i}\left\lceil\alpha_{i}\right.$ actually should be the function $g(\delta)=f_{i}(\delta) \uparrow \alpha_{i}$.) Note that a simple construction using the $F_{1}^{\alpha_{j}}$,s shows that $f_{i+1}$ as above does exist. So we get that $f_{\lambda}$ is a condition for limit $\lambda$ and $f_{\operatorname{cof}(\alpha)}$ is as desired.

If $\operatorname{cof}(\alpha) \leq \kappa$ we define $F_{n}^{\alpha}(x, \kappa, p)$ to be the least $q \leq_{\kappa} p$ (in the given 
$\Delta_{1}^{A}$ well-ordering of $\left.\langle V, A\rangle\right)$ such that $q\left\lceil\alpha_{i} \leq_{\kappa} F_{n}^{\alpha_{i}}\left(x, \kappa, p\left\lceil\alpha_{i}\right)\right.\right.$ for each $\alpha_{i}$ in a fixed $\operatorname{cof}(\alpha)$-sequence cofinal in $\alpha$. If $\kappa<\operatorname{cof}(\alpha)<\alpha$ and $\operatorname{cof}(\alpha)$ is not the successor of a regular cardinal then we obtain $F_{n}^{\alpha}(x, \kappa, p)$ by first choosing $q_{0} \leq_{\operatorname{cof}(\alpha)} p$ so that $q_{0}\left\lceil\alpha_{i} \leq_{\operatorname{cof}(\alpha)} F_{n}^{\alpha_{i}}\left(x, \operatorname{cof}(\alpha), p\left\lceil\alpha_{i}\right)\right.\right.$ for each $\alpha_{i}$ and then $q_{1} \leq_{\kappa} q_{0}$ so that $q_{1} \uparrow \operatorname{cof}(\alpha) \leq_{\kappa} F_{n}^{\operatorname{cof}(\alpha)}\left(x, \kappa, q_{0} \uparrow \operatorname{cof}(\alpha)\right)$. If $\kappa \leq \lambda<\lambda^{+}=\operatorname{cof}(\alpha)<\alpha$ with $\lambda$ regular then we choose $q_{0}, q_{1}$ as above and then $q_{2} \leq_{\lambda} q_{1}$ so that $q_{2} \uparrow \beta \leq_{\lambda} F_{n}^{\beta}\left(x, \lambda, q_{1} \uparrow \beta\right)$ for each $\beta$ such that $\operatorname{cof}(\alpha) \leq \beta$ and $q_{1} \uparrow \beta \forall \forall_{\beta} q_{1}(\beta)$ is the $\emptyset$-function below $\operatorname{cof}(\alpha)$. Finally if $\kappa<\alpha$ and $\alpha$ is regular then choose $q_{0}$ as above $\left(\alpha_{i}=i\right)$ and then $q_{1} \leq_{\kappa} q_{0}$ so that $q_{1}\left\lceil\beta \leq_{\kappa} F_{n}^{\beta}\left(x, \kappa, q_{0}\lceil\beta)\right.\right.$ where $\beta<\alpha$ is least so that $\beta \leq \beta^{\prime} \longrightarrow q_{0} \uparrow \beta^{\prime} \Vdash_{\beta^{\prime}} q_{0}\left(\beta^{\prime}\right)$ is the $\emptyset$-function below $\alpha^{+}$. Our construction guarantees that if $q=F_{n}^{\alpha}(x, \kappa, p)$ and $\beta<\alpha$ then for some $\kappa^{\prime} \geq \kappa$ and $q^{\prime}, q\left\lceil\beta \leq_{\kappa} q^{\prime} \leq_{\kappa^{\prime}} F_{n}^{\beta}\left(x, \kappa^{\prime}, p^{\prime}\right)\right.$ for some $p^{\prime} \leq_{\kappa} p\lceil\beta$. The latter is used to verify $\kappa$-Definable Closure when $\alpha$ is regular. (The other cases are straightforward, using our extra hypothesis about the $F_{n}$ 's stated at the start of the proof.)

Finally we must establish the second part of $\kappa$-Density Reduction for $P_{\alpha}^{*}$. (The first part is easy if $\operatorname{cof}(\alpha) \leq \kappa$ and otherwise follows inductively.) First suppose that $\alpha<\kappa^{+}$and choose an increasing cofinal $\alpha_{0}<\alpha_{1}<\ldots$ of ordertype $\operatorname{cof}(\alpha)$. Given $p \in P_{\alpha}^{*}$ and $A$-definable open dense $D$, use the $F_{n}^{\alpha_{i}}$ functions to successively extend $p\left\lceil\alpha_{i}\right.$ producing $q \leq_{\kappa} p$ such that for each $i<\operatorname{cof} \alpha, q\left\lceil\alpha_{i}\right.$ reduces $D^{\alpha_{i}}$ below $\kappa^{+}$to size $\leq \kappa$, where $D^{\alpha_{i}}=\left\{r\left|\alpha_{i}\right| r \in D\right\}$. Now successively $\leq_{\kappa}$-extend $q=q_{0}$ to $q_{1}, q_{2}, \ldots$ so that for each $\gamma$ there is $x_{\gamma+1}$ defined on Card $\cap \kappa^{+}$so that $x_{\gamma+1} \cup q_{\gamma+1}$ is an element of $D$ yet is incompatible with each $x_{\gamma^{\prime}+1} \cup q_{\gamma^{\prime}+1}$ for $\gamma^{\prime}<\gamma$. But for each $i$ there must be a stage $\gamma_{i}<\kappa^{+}$such that for $\gamma \geq \gamma_{i},\left(x_{\gamma+1} \cup q_{\gamma+1}\right) \uparrow \alpha_{i}$ is compatible with some $\left(x_{\gamma^{\prime}+1} \cup q_{\gamma^{\prime}+1}\right) \uparrow \alpha_{i}$ where $\gamma^{\prime}<\gamma_{i}$, since $q\left\lceil\alpha_{i}\right.$ reduces $D^{\alpha_{i}}$ below $\kappa^{+}$to size $\leq \kappa$. Let $\gamma=\cup\left\{\gamma_{i} \mid i<\operatorname{cof} \alpha\right\}<\kappa^{+}$. Then $q_{\gamma+1}$ is undefined so some $q_{\gamma^{\prime}}, \gamma^{\prime}<\kappa^{+}$reduces $D$ below $\kappa^{+}$, to size $\leq \kappa$.

Now suppose that $\alpha \geq \kappa^{+}$. We may assume that $\alpha=\kappa^{+}$as short diagonal supports requires that $p \in P_{\alpha}^{*}$ are trivial below $\kappa^{+}$on all but fewer than $\kappa$ coordinates, all below $\kappa^{+}$. But note that we can assume that conditions in $D$ when restricted to Card $\cap \kappa^{+}$belong to $H_{\kappa^{+}}$and therefore can choose $q \leq_{\kappa} p$ and $\alpha_{o}<\kappa^{+}$of cofinality $\kappa$ such that the conditions in $D$ which are trivial below $\kappa^{+}$on coordinates $\geq \alpha_{0}$ form a set predense below $q$. If we extend $q$ to $q_{0} \leq_{\kappa} q$ reducing $D^{\alpha_{0}}$ below $\kappa^{+}$to size $\leq \kappa$, then $q_{0}$ in fact reduces $D$ below $\kappa^{+}$to size $\leq \kappa$.

There are some important examples of cofinality-preserving class forcings that are not stratified. Instead they may obey $\Delta$-stratification, which we now consider. 
Definition. $P$ is $\Delta$-stratified if it obeys the definition of stratified where (b), (c) are restricted to successor cardinals and in addition: whenever $0<n \in \omega$ and $\kappa$ is inaccessible, $p_{0} \geq p_{1} \geq \ldots$ a $\Pi_{n}^{A}$ (in parameters from $\lambda \cup\{x\})$ sequence of length $\lambda \leq \kappa$ and for each $i<\lambda, p_{i+1} \leq_{\kappa_{i}} F_{n}\left(x, \kappa_{i}, p_{i}^{\prime}\right)$ for some $p_{i}^{\prime} \leq_{\kappa_{i}} p_{i}$ and regular $\kappa_{i} \geq \aleph_{i+1}$, there is a single $p$ s.t. $p \leq \aleph_{i+1} p_{i}$ for each $i$.

$A$ is a strong witness to the $\Delta$-stratification of $P$ if it obeys the definition of strong witness to stratification when restricted to successor cardinals. A $\Delta$-stratified iteration is just like a stratified iteration but with stratified replaced by $\Delta$-stratified everywhere. Such an iteration $\left\langle P_{i} \mid i<\alpha\right\rangle$ has long diagonal supports if for $j<\alpha, p \in P_{j}$ and successor cardinals $\kappa$, $\left\{i<j \mid p\lceil i \| \forall \forall \gamma \leq \kappa, p(i)(\gamma)=\emptyset\}\right.$ is a subset of $\kappa^{+}$of size $<\kappa$ and for inaccessible $\kappa \leq j,\left\{\bar{\kappa}<\kappa \mid\right.$ For some $\bar{\kappa} \leq j^{\prime}<j, p\left\lceil j^{\prime} \forall j_{j^{\prime}} p\left(j^{\prime}\right)\right.$ is $\emptyset$ at $\left.\bar{\kappa}\right\}$ is nonstationary in $\kappa$ (and these are the only support restrictions).

Theorem 4. Suppose that $P$ is $\Delta$-stratified. Then $P$ preserves $Z F C$ (relative to the class $A$ witnessing $\Delta$-stratification), cofinalities and the $G C H$.

Proof. As in the proof of Theorem 1, using $\Delta$-stratification at $\kappa$ and stratification at $\bar{\kappa}^{+}<\kappa$, when $\kappa$ is inaccessible.

$\Delta$-Stratification Theorem. Suppose $\left\langle P_{i}\right| i\langle\alpha\rangle$ is a $\Delta$-stratified iteration with long diagonal supports and $G C H$ holds. Then $P_{\alpha}$ is isomorphic to a $\Delta$-stratified forcing (definably relative to a class $A$ witnessing $\Delta$-stratification).

Proof. We follow the proof of the Stratification Theorem. Note that Theorem 3 still applies by the Remark following the proof of that theorem. We proceed by induction on $\alpha$. For successor $\alpha$ our earlier proof still shows that (b), (c) hold at successor cardinals. For $\Delta$-stratification at an inaccessible $\kappa$, use $\Delta$-stratification for $P_{\beta}^{*}(\alpha=\beta+1), \emptyset_{\beta} \Vdash_{\beta} \Delta$-stratification for $\mathbb{Q}_{\beta}^{*}$ to obtain $\bar{p} \uparrow \beta \leq_{\aleph_{i+1}} p_{i} \uparrow \beta$ for each $i, \bar{p} \uparrow \beta \Vdash_{\beta}$ there is $\bar{p}(\beta) \leq_{\aleph_{i+1}} p_{i}(\beta)$ for each $i$ and then $\leq_{\kappa^{+}}$extend $\bar{p} \uparrow \beta$ to $p\lceil\beta$ so that for some term $p(\beta)$, $p\left\lceil\beta \Vdash_{\beta} p(\beta) \leq_{\aleph_{i+1}} p_{i}(\beta)\right.$ for each $i$, using $\kappa^{+}$-Density Reduction. Then $p \leq_{\aleph_{i+1}} p_{i}$ for each $i$ is as desired.

When $\alpha$ is a limit ordinal we define $P_{\alpha}^{*}$ as before and first show that

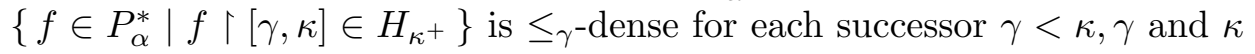
in Card. We do this by induction on $\kappa$, noting that we may assume it holds for $P_{\beta}^{*}, \beta<\alpha$. Using (long) diagonal supports we may assume that either $\alpha=\kappa$ is inaccessible or $\operatorname{cof}(\alpha)<\kappa$. If $\operatorname{cof}(\alpha)$ is a successor or $\operatorname{cof}(\alpha)^{+}<\kappa$ then the old argument can be applied, using $\operatorname{cof}(\alpha)$-Definable Closure or $\operatorname{cof}(\alpha)^{+}$-Definable Closure applied to $P_{\alpha_{i}}^{*}, \alpha_{i}<\alpha$. So we may assume that either $\alpha=\kappa$ is inaccessible or $\operatorname{cof}(\alpha)^{+}=\kappa$ where $\operatorname{cof}(\alpha)$ is inaccessible. 
In the latter case we choose a cofinal $\operatorname{cof}(\alpha)$ sequence $\alpha_{0}<\alpha_{1} \ldots$ and successively $\leq_{\gamma}$-extend our given $f=f_{0}$ to $f_{1}, f_{2}, \ldots$ in $\operatorname{cof}(\alpha)$ steps so that $\left(f_{i+1}\left\lceil\alpha_{i}\right)(\kappa) \in H_{\kappa^{+}}\right.$and $f_{i+1}\left\lceil\alpha_{j} \geq_{\aleph_{j+1}} F_{1}^{\alpha_{j}}\left(x, \aleph_{j+1} \cup \gamma, f_{i}\left\lceil\alpha_{j}\right)\right.\right.$ for all $j \leq i, x=\left\langle f, \gamma, \kappa,\left\langle\alpha_{i} \mid i<\operatorname{cof}(\alpha)\right\rangle\right\rangle$. Note that by induction we may extend $g=f_{\operatorname{cof}(\alpha)}$ so that $g\left\lceil[\gamma, \operatorname{cof}(\alpha)] \in H_{\kappa}\right.$, as desired. Finally if $\alpha=\kappa$ is inaccessible use Definable Closure to successively $\leq_{\gamma}$-extend $f=f_{0}$ to $f_{1}, f_{2}, \ldots$ in $\kappa$ steps choosing a continuous cofinal $\kappa_{0}<\kappa_{1}<\ldots$ below $\kappa$ such that $f_{i+1} \uparrow\left(\kappa_{i}, \kappa_{i+1}\right)$ belongs to $H_{\kappa_{i+1}^{+}}$and $f_{i+1}\left(\kappa_{i}\right)=\emptyset$ for all $i$, using the fact that $\{\gamma<\kappa \mid f(\gamma) \neq \emptyset\}$ is nonstationary in $\kappa$. Then $f_{\kappa}$ is as desired.

If $\operatorname{cof}(\alpha) \leq \kappa$ or $\alpha$ is a successor cardinal or $\operatorname{cof}(\alpha)$ is neither inaccessible nor the successor of an inaccessible then we define $F_{n}^{\alpha}(x, \kappa, p)$ as in the stratified case. If $\operatorname{cof}(\alpha)>\kappa$ is inaccessible then let $\alpha_{0}<\alpha_{1}<\ldots$ be a cofinal $\operatorname{cof}(\alpha)$-sequence so that $\alpha_{j} \geq \aleph_{j+2} \cup \kappa$ for each $j<\operatorname{cof}(\alpha)$ and let $F_{n}^{\alpha}(x, \kappa, p)$ be a lower bound of $p=p_{0}, p_{1}, \ldots$ where $p_{j+1}$ is least so that $p_{j+1} \uparrow \alpha_{j^{\prime}} \leq_{\kappa \cup \aleph_{j+1}} F_{n}^{\alpha_{j^{\prime}}}\left(x, \kappa \cup \aleph_{j+1}, p_{j} \uparrow \alpha_{j^{\prime}}\right)$ for all $j^{\prime} \leq j$. If $\kappa \leq \lambda<\lambda^{+}=\operatorname{cof}(\alpha)<\alpha, \lambda$ inaccessible then similarly modify the earlier definition of $q_{2}$, enumerating the relevant $\beta$ 's in $\lambda$ steps.

$\kappa$-Density Reduction for successor $\kappa$ follows just as in the stratified case. $\Delta$-stratification also follows as our construction implies that if $p_{i+1}$ $\leq_{\aleph_{i+1}} F_{n}^{\alpha}\left(x, \aleph_{i+1}, p_{i}\right)$ for $i<\kappa(\kappa$ inaccessible $)$ then for cofinally many $\alpha^{\prime}<\alpha, p_{i+1}\left\lceil\alpha^{\prime} \leq_{\aleph_{i^{\prime}+1}} F_{n}^{\alpha^{\prime}}\left(x, \aleph_{i^{\prime}+1}, p_{i}\left\lceil\alpha^{\prime}\right)\right.\right.$ for each $i$ (and some $i^{\prime} \geq i$ depending on $\left.\alpha^{\prime}, i\right)$. Also if $\beta<\alpha$ and $p \leq_{\kappa} q$ in $P_{\beta+1}^{*}, p$ at $\beta=q$ at $\beta$ then $F_{n}^{\beta+1}(x, \kappa, p)$ at $\beta$ equals $F_{n}^{\beta+1}(x, \kappa, q)$ at $\beta$. So given $p_{0}, p_{1}, \ldots$ of length $\lambda$ as in the hypothesis of $\Delta$-stratification at $\kappa$ for $P_{\alpha}^{*}$, we can obtain the desired lower bound $p$ by choosing $q \uparrow \beta+1$ to be a lower bound for $\left\langle p\left\lceil\beta \cup\left\{\left\langle\beta, p_{i}\right.\right.\right.\right.$ at $\left.\left.\beta\right\rangle\right\}|i<\lambda\rangle$ and taking $p(\beta)=q(\beta)$.

\section{Examples.}

(a) Jensen coding (Beller-Jensen-Welch [82]) is equivalent to a $\Delta$ stratified forcing. It is dense to have $p(0) \neq \emptyset$ and restricted to such conditions (together with the $\emptyset$ conditions) condition (a) is satisfied. (We must reindex though: $p(\kappa)=p\left(\kappa^{+}\right)$in Jensen's sense.) The first part of (b) is clear at successor $\kappa$ and the second part is one of Jensen's key lemmas (Lemma 3.8). For (c) we take $F_{n}(x, \kappa, p)$ to be the least $q \leq_{\kappa} p$ such that for $\lambda \leq \gamma \in \operatorname{Dom}(p), \gamma \in \Sigma_{n-1}^{A} \operatorname{Hull}(\gamma \cup\{x\}),(q)_{\gamma}$ meets all predense $D$ on $P_{\gamma}$ in $\Sigma_{n-1}^{A}$ Hull $(\gamma \cup\{x, p\})$. Jensen's lemmas show that such a $q$ exists and that (c) is satisfied. (Theorem 3.2. One can assume that all the $\kappa_{i}$ 's are equal by looking at their lim inf). The extra $\Delta$-stratification condition also follows from Jensen's work (Theorem 3.2.).

(b) The modification of Jensen coding in Friedman [94A] is equivalent 
to a forcing that is both stratified and $\Delta$-stratified. It is densely embeddable in the forcing defined in the same way (after reindexing as in (a)) but where at limit cardinals $\kappa$, we allow $p \uparrow \kappa$ to code only an initial segment of $p_{\kappa}$ (and belong to the coding structure for that initial segment). This allows one to prove (c) at inaccessibles (Lemma, 2.4, 2.6). The thinning that was done there in the limit coding enables one to prove (b) at inaccessibles (Lemma 2.1).

(c) The modification of Jensen coding in Friedman [94B] is stratified. The proof of (b) at inaccessibles uses the fact that conditions have Easton domains (see the proof of Lemma 4).

(d) Easton forcing (see Easton [70]) where a Cohen set is added to each regular cardinal via an Easton product is stratified. (Take $F_{n}(x, \kappa, p)=p$.) If, instead, the full product is used but only at successor cardinals (no restriction on the domains of conditions) then $\Delta$-stratification is obtained (but (b) will hold only at successors). Without the restriction to successor cardinals one has a "hybrid" forcing that is neither stratified nor $\Delta$-stratified. Iterating it would require use of "mixed support."

(e) The forcing of Friedman [90] is a mixture of Jensen-style and Easton-style forcing. It is equivalent to a stratified forcing, provided one of the stratified modifications of Jensen coding (see (b), (c) above) is used (Lemmas 9,16).

(f) Backwards Easton forcings with Easton support (see Jech [78], section 36) are stratified provided at regular $\kappa$ one uses a $\kappa^{+}$-CC forcing of size $\leq \kappa^{+}$(Lemmas 36.4, 36.5).

\section{References}

[82] A. Beller, R. Jensen and P. Welch, Coding The Universe, Cambridge University Press, 1982.

[70] W. Easton, Powers of regular cardinals, Annals of Mathematical Logic 1 (1970), 139-178.

[90] S. Friedman, The $\Pi_{2}^{1}$-Singleton conjecture, Journal of the AMS 3 (1990), 771-791.

[94A] _ A simpler proof of Jensen's coding theorem, Annals of Pure and Applied Logic (to appear).

[94B] _ A short proof of Jensen's coding theorem assuming not 0\# (to appear).

[78] T. Jech, Set Theory, Academic-Press, 1978.

Department of Mathematics, M.I.T., Cambidge, M A 02139

E-mail address: sdf@math.mit.edu 